Document downloaded from:

http://hdl.handle.net/10251/119374

This paper must be cited as:

Zamorano -López, N.; Moñino Amoros, P.; Borrás Falomir, L.; Aguado García, D.; Barat, R.; Ferrer, J.; Seco Torrecillas, A. (2018). Influence of Food Waste addition over microbial communities in an Anaerobic Membrane Bioreactor plant treating urban wastewater. Journal of Environmental Management. 217:788-796. https://doi.org/10.1016/j.jenvman.2018.04.018

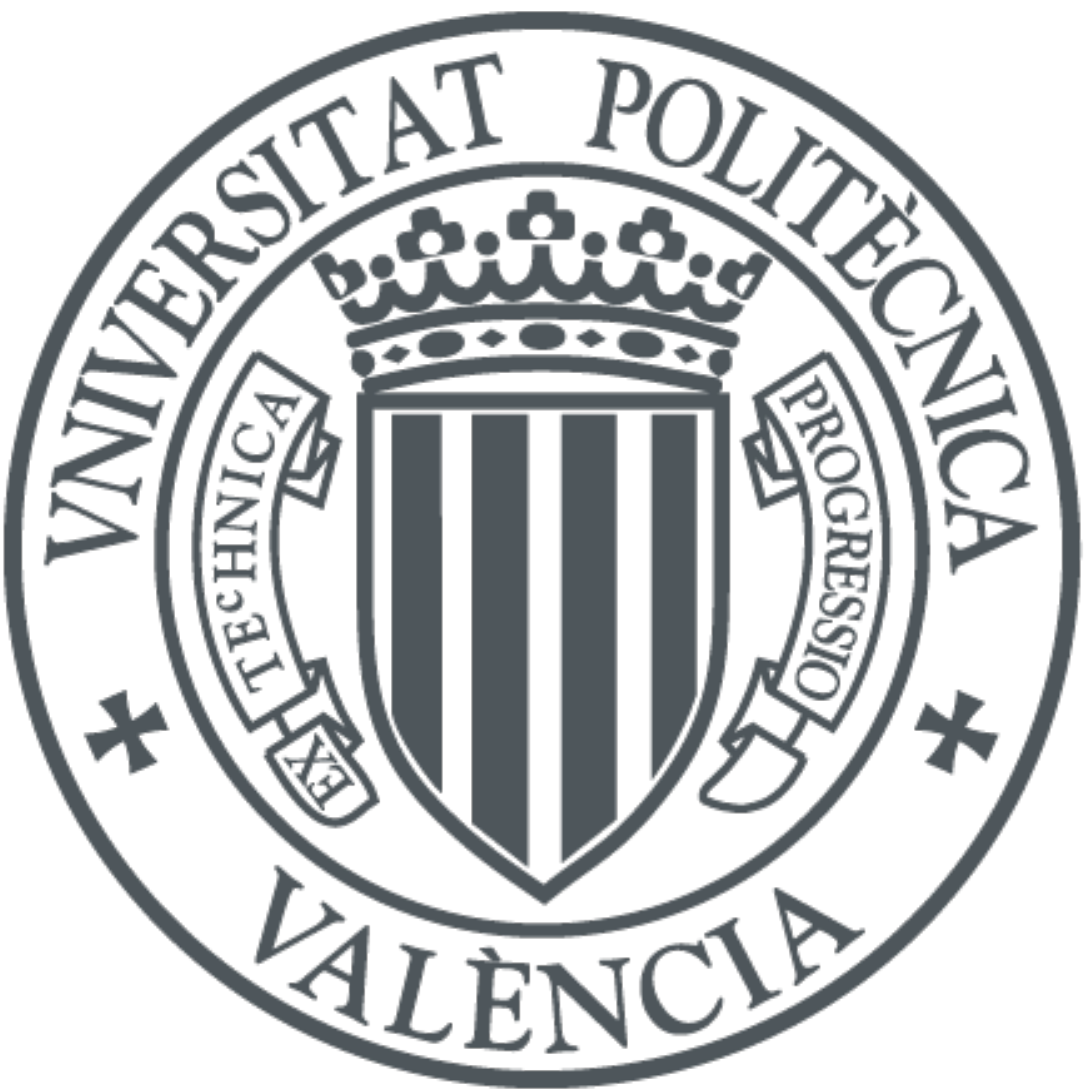

The final publication is available at

https://doi.org/10.1016/j.jenvman.2018.04.018

Copyright Elsevier

Additional Information 


\title{
Influence of Food Waste addition over microbial communities in an Anaerobic Membrane Bioreactor plant treating urban wastewater
}

N. Zamorano-López*, P. Moñino**, L. Borrás*, D. Aguado**, R. Barat**, J. Ferrer**, A. Seco*

*CALAGUA - Unidad Mixta UV-UPV, Departament d’Enginyeria Química, Universitat de València, Avinguda de la Universitat s/n, 46100 Burjassot, Valencia, Spain.

**CALAGUA - Unidad Mixta UV-UPV, Institut Universitari d'Investigació d'Enginyeria de l'Aigua i Medi Ambient - IIAMA, Universitat Politècnica de Valencia, Camí de Vera s/n, 46022 Valencia, Spain.

Corresponding authors: Zamorano-Lopez, Nuria (nuria.zamorano@uv.es), CALAGUA - Unidad Mixta UV-UPV, Departament d'Enginyeria Química, Universitat de València, Avinguda de la Universitat s/n, 46100 Burjassot, Valencia, Spain

\begin{abstract}
Notorious changes in microbial communities were observed during and after the joint treatment of wastewater with Food Waste (FW) in an Anaerobic Membrane Bioreactor (AnMBR) plant. The microbial population was analysed by high-throughput sequencing of the 16S rRNA gene and dominance of Chloroflexi, Firmicutes, Synergistetes and Proteobacteria phyla was found. The relative abundance of these potential hydrolytic phyla increased as a higher fraction of FW was jointly treated. Moreover, whereas Specific Methanogenic Activity (SMA) rose from 10 to $51 \mathrm{~mL} \mathrm{CH}_{4} \mathrm{~g}^{-1} \mathrm{VS}$, Methanosarcinales order increased from 34.0\% over $80.0 \%$ of total Archaea, being Methanosaeta the dominant genus. The effect of FW over AnMBR biomass was observed during the whole experience, as methane production rose from 49.2 to $144.5 \mathrm{~L} \mathrm{CH}_{4} \cdot \mathrm{kg}^{-}$ ${ }^{1}$ influent COD. Furthermore, biomethanization potential was increased over $82 \%$ after the experience. AnMBR technology allows the established microbial community to remain in the bioreactor even after the addition of FW, improving the anaerobic digestion 1
\end{abstract}


of urban wastewater.

\section{Keywords}

anaerobic digestion; AnMBR; biogas; food waste; Illumina; microbial community.

\section{Introduction}

The anaerobic digestion (AD) of waste has become popular due to its environmental sustainability, as it not only reduces waste production, but also enables bioenergy production (Mao et al., 2015). Methane-rich biogas is produced during the degradation of organic matter through different microbiologically-controlled stages, such as hydrolysis, fermentation, acidogenesis and methanogenesis.

An Anaerobic Membrane Bioreactor (AnMBR) decouples the hydraulic retention time (HRT) from the sludge retention time (SRT), allowing the application of AD to low strength wastewaters treatment, such as urban wastewater (WW). This technology has a suitable effect over AD of WW even when treating urban influents with high concentration of sulfates, which can lead to low methane yields (Giménez et al., 2011). Moreover, the use of membrane technology provides full biomass retention in the digester with reasonable digester volumes, enhancing the heterogeneity of the system and improving domestic WW treatment (Smith et al., 2015).

The AD of food waste (FW) can also contribute to reducing the amount of organic wastes sent to landfills, as required by the European 1999/31/CE Directive. Also, this enhanced version of $\mathrm{AD}$ can be a proper way for food disposal and comply with the European 98/2008/CE Directive. Incorporating the FW into the WW influent for joint treatment via AD can improve energy recovery and has other benefits, such as savings in municipal solid waste transportation, reducing fossil fuel consumption and landfill volumes (Kujawa-Roeleveld et al., 2006). The small carbon footprint of food waste disposers and associated water consumption have been reviewed by Mattsson and co-workers (2015). 
Several studies have addressed the treatment of FW (Fisgativa et al., 2017; Vrieze et al., 2015). However, only a few have focused on AnMBR (Galib et al., 2016) to convert this organic enhanced waste stream into energy.

Microbial population in $\mathrm{AD}$ processes provides valuable information and must be considered jointly with process parameters monitoring (Tan et al., 2016). A heterogeneous pool of molecular biological tools can be used to characterize microbial populations. Next generation sequencing (NGS) has especially changed the study of microbial ecology in complex environments such as anaerobic digesters, being Illumina the most applied sequencing technique, due to its reduced cost and the useful information it provides on the microbial population. High-throughput sequencing of biomarkers such as the 16S rRNA gene is a valuable tool for the identification and quantification of key microbial groups in AD (Bartram et al., 2011; Degnan and Ochman, 2012; Vanwonterghem et al., 2014).

Most previous studies have focused on the methanogenic population of anaerobic digesters, due to its importance in the operational efficiency and energy recovery (Alvarado et al., 2014; Wilkins et al., 2015). However, a global overview of the microbial communities, considering both the Archaea and Bacteria domains, is needed to understand the implications of these microorganisms in limiting $\mathrm{AD}$ steps such as hydrolysis and fermentation. Thus, besides monitoring performance parameters, a thorough analysis of microbial populations with the new molecular tools is needed to better understand $\mathrm{AD}$ seeking the improvement of this process management (Carballa et al., 2015).

In this study, a joint treatment of FW and urban WW has been performed in an AnMBR demonstration plant, generating high energy recovery yields in terms of methane and biogas production (Moñino et al., 2017). The notorious improve of the AD of urban WW 3 
once the FW addition was over, suggested that microbial population established during the experience was more efficient than the previous one established. Hence, microbial insights of the AnMBR demonstration plant are here explored, revealing the remarkable influence of FW substrate and membrane technology over microbial populations.

\section{Materials and methods}

\subsection{Demonstration plant}

The AnMBR demonstration plant used in this study is situated in the Carraixet WWTP, in Alboraya (València, Spain) (see the process flow diagram in Figure 1). The influent for this plant is taken from the pre-treatment of the Carraixet WWTP, after screening and removal of grit and grease. Then, it is treated in a $0.5 \mathrm{~mm}$ screen rotofilter, homogenised in the regulation tank (RT) and pumped into a $1.3 \mathrm{~m}^{3}$ anaerobic reactor $\left(0.4 \mathrm{~m}^{3}\right.$ headspace volume). This digester is connected to two external membrane tanks of $0.8 \mathrm{~m}^{3}$ total volume each $\left(0.2 \mathrm{~m}^{3}\right.$ head-space volume), set in parallel, which allow to do chemical membrane cleaning or another maintenance operation needed without interrupting the biological process performance. In the membrane tanks, vacuum filtration is applied to obtain the effluent, which is stored in a Clean-in-Place tank. Sludge is continuously recycled from the anaerobic reactor to the membrane tanks and the SRT is controlled by purging a fraction of the sludge from the anaerobic reactor intermittently during the day. A commercial food waste disposer and a $0.5 \mathrm{~mm}$ space screen rotofilter are used for the pre-treatment of the FW, which is stored in a co-substrate tank (CT) with a usable volume of $0.180 \mathrm{~m}^{3}$ and is also connected to the anaerobic reactor. A three-way valve alternates wastewater and FW inputs from the RT or CT, respectively. 


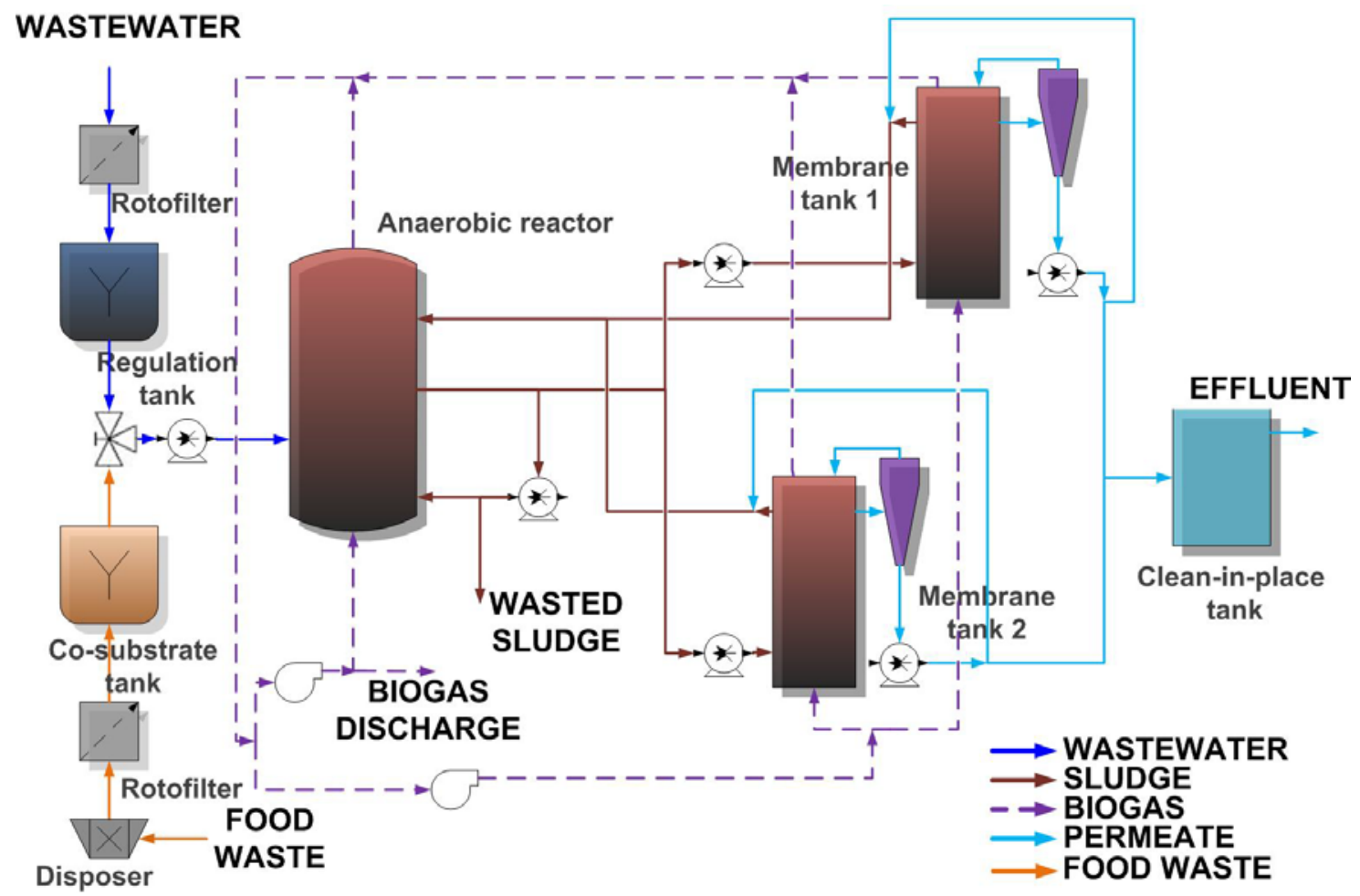

Figure 1. AnMBR demonstration plant process flow diagram.

The FW fraction is supplied according to the Penetration Factor (PF) established, which is defined as the percentage of households using food waste disposers. Two scenarios were evaluated, assuming that $40 \%$ or $80 \%$ of the population were grinding the food FW. These scenarios were explored as they might be feasible in small areas where household food waste disposers can be implemented. According to the national plan for waste management (PNIR 2008-2015), a mean value of $0.63 \mathrm{~kg} \mathrm{FW} \cdot \mathrm{hab}^{-1} \cdot \mathrm{d}^{-1}$ is generated in Spain. The Statistical National Institute of Spain reported in 2010 an urban wastewater generation of $282.4 \mathrm{~L} \cdot \mathrm{hab}^{-1} \cdot \mathrm{d}^{-1}$ in 2010 (last available data). From this volume 225.92 $\mathrm{L} \cdot \mathrm{hab}^{-1} \cdot \mathrm{d}^{-1}$ (an $80 \%$ approximately) is considered to have a domestic origin. Experimental results determined that a FW and WW mixture of $2.52 \mathrm{~L} \cdot \mathrm{hab}^{-1} \cdot \mathrm{d}^{-1}$ is generated during FW grinding in household disposers. Hence, a resulting ratio of $11.2 \mathrm{~mL}$ of grinded FW per L of WW was fed to the pilot plant: 4.48 and $8.96 \mathrm{~mL}$ of $\mathrm{FW}$ per $\mathrm{L}$ of $\mathrm{WW}$, representing a $40 \%$ and $80 \%$ PF scenario, respectively.

\subsection{Operational conditions}

5 
Four different pseudo steady-state periods (Table 1), determined after stabilising solids concentration and methane production in the AnMBR, were selected for microbial community analysis. In Periods 2 and 3, the AnMBR treated both FW and wastewater substrates at different PF (40 and 80\%, respectively). In the remaining periods, only wastewater was treated. Period 1 was prior to the joint treatment and Period 4 was after FW addition, when a new pseudo steady-state had been reached.

Table 1. Operational conditions of each pseudo steady-state period studied in the AnMBR plant.

\begin{tabular}{lcccc}
\hline & Period 1 & Period 2 & Period 3 & Period 4 \\
\hline SRT (d) & $42 \pm 2$ & $70 \pm 11$ & $69 \pm 6$ & $70 \pm 2$ \\
T $\left({ }^{\circ} \mathrm{C}\right)$ & $25 \pm 2$ & $28 \pm 1$ & $27 \pm 1$ & $28 \pm 3$ \\
HRT $(\mathrm{h})$ & $30 \pm 4$ & $22 \pm 6$ & $24 \pm 6$ & $22 \pm 4$ \\
PF $(\%)^{*}$ & 0 & 40 & 80 & 0 \\
Treatment flow $\left(\mathrm{L} \cdot \mathrm{d}^{-1}\right)$ & $1630 \pm 154$ & $2223 \pm 516$ & $2038 \pm 549$ & $2223 \pm 359$
\end{tabular}

$*$ PF was defined as the percentage of households that use food waste disposers.

\subsection{Biological process monitoring}

Influent, effluent and AnMBR reactor samples were collected twice a week to monitor the biological process. Volatile Solids (VS), COD, sulphide and sulfate concentrations were determined according to Standard Methods (APHA, 2005). Methane production was recorded and dissolved methane in the effluent was calculated by Henry's Law, as described in Giménez et al. (2012). Specific methanogenic activity (SMA) tests were carried out for each period using the Automatic Methane Potential Test System (AMPTS) [Bioprocess Control, Sweden] and performed as described in Ozgun et al. (2015).

\subsection{Sample collection and DNA extraction}

Sludge samples were collected from the AnMBR at each period (see Table 1) and were immediately stored in $1 \mathrm{~mL}$ cryotubes at $-20^{\circ} \mathrm{C}$ to characterize the microbial population involved in the AD process. Extraction of DNA was performed in an E.Z.N.A Soil DNA Kit (Omega-Biotek), according to the manufacturer's protocol but with minor 
modifications to improve the DNA yield (data not shown): (i) incubation time was increased from 10 to 20 minutes at $70^{\circ} \mathrm{C}$ and (ii) the second incubation was at $95^{\circ} \mathrm{C}$ for 5 minutes during the cell lysis stage. Extractions were performed from $1 \mathrm{~mL}$ of homogenized sludge. A Nanodrop 2000 spectrophotometer (Thermo Scientific) was used to determine the concentration and purity of DNA through the absorbance measured at wavelengths of 260, 230 and $280 \mathrm{~nm}$. In order to avoid contamination by RNA, humic acids or other compounds, only sequences with an A260/230 ratio between 2.0 and 2.2 and an A260/280 ratio over 1.8 were sequenced.

\subsection{Illumina amplicon sequencing}

A set of libraries from the v4 hyper-variable region of the 16S rRNA gene were prepared according to the procedure described in Caporaso et al. (2011). Universal prokaryotic indexed primers 515F (5'-GTGCCAGCMGCCGCGGTAA-3') and 806R (5’GGACTACHVGGGTWTCTAAT-3'), were used for this purpose according to the following amplification conditions: denaturing stage at $95^{\circ} \mathrm{C}$ during 30 seconds, 28 cycles of 30 seconds steps at 95,55 and $72{ }^{\circ} \mathrm{C}$, successively; and final elongation stage at $72{ }^{\circ} \mathrm{C}$ during 5 minutes. The concentration of DNA in selected samples was determined in a Qubit 3.0 fluorometer (Life Technologies) and $0.2 \mathrm{ng} / \mu \mathrm{L}$ of each DNA sample were used for library preparation with indexed primers. The resulting amplicons were multiplexed in a Nextera XT Index Kit (Illumina) and sequencing was performed according to the Illumina manufacturer's protocol in a MiSeq reagent kit V3, on a MiSeq sequencer in a 2x300 bp paired-end run, in genomic department of the Fundación para el Fomento de la Investigación Sanitaria y Biomédica de la Comunidad Valenciana (FISABIO).

\subsection{Illumina processing data and statistics}

Raw data retrieved from Illumina sequencing after barcode and index removal was sequentially processed through the following pipeline: first, the prinseq-pl algorithm 
(Schmieder and Edwards, 2011) was applied at the trimming stage, within a qualitythreshold of 30 and a window length of $12 \mathrm{bp}$. The trimmed paired-end reads were merged together with default parameters of fastq-join (Aronesty, 2011), and checked for chimeras within the UCHIME algorithm (Edgar et al., 2011). Non-chimeric sequences were classified up to genus level, applying a confidence threshold of 0.8 , in the Ribosomal Database Project's Classifier tool release 2.11 (Cole et al., 2009). R-software and the Vegan v.2.3-1 package (Oksanen et al., 2016) were used to estimate relative abundances between samples and to calculate diversity and evenness indexes: Shannon-Wiener and Simpson (expressed as inverse Simpson for a better comparison with the Shannon-Wiener index). Raw sequences have deposited as follows: Database: BioProject (PRJNA339420, samples SRS2046188, SRS2046189, SRS2046190 and SRS2046191).

\section{Results and discussion}

\subsection{AnMBR demonstration plant performance.}

The FW treated in this study was mainly characterized by high carbohydrate content and a remarkable presence of polysaccharides, according to the following frequency of occurrence: rice (88\%), fruit remains and peel (80\%), potatoes (68\%), bread (64\%), pasta (56\%), seafood (52\%), cooked vegetables (44\%), chicken (32\%), salad (16\%), fish (16\%), pork (8\%) and beef (8\%). The exhaustive characterization performed in Moñino et al. (2016), showed that ground FW presents high COD (100 times higher than the average concentration in WW) and small size (90\% of the particles under $0.5 \mathrm{~mm})$, allowing a significant part of the FW to reach the AnMBR despite the restrictive pre-treatment of 0.5 mm sieve. This substrate can be more easily hydrolysed than WW, as demonstrated by previous assays which shown $72 \%$ of anaerobic biodegradability, leading to increase the production of volatile fatty acids (VFAs) and other fermentation by-products in the digester. The higher production of compounds like acetate or hydrogen enhances the 
system's methane production potential, as they are substrates available for MA, whose can finally reduce them to methane.

Four pseudo steady-state periods were defined according to the stabilised concentration of VS in the AnMBR (see Table 2). The COD and sulfate concentrations for each period are also shown in Table 2 for WW, FW and the total concentration of the influent. It is remarkable that, due to the FW addition, the COD concentration in the influent increases while the sulfate concentration remains in the same range, in Periods 2 and 3. Consequently, the $\mathrm{COD} / \mathrm{S}-\mathrm{SO}_{4}$ ratio was increased in the co-treatment periods.

Table 2. Performance and biological process monitoring of AnMBR plant.

\begin{tabular}{|c|c|c|c|c|}
\hline & Period 1 & Period 2 & Period 3 & Period 4 \\
\hline \multicolumn{5}{|l|}{ Influent characteristics } \\
\hline FW COD $\left(\mathrm{mg} \mathrm{COD} \cdot \mathrm{L}^{-1}\right)$ & & $69455 \pm 20130$ & $71872 \pm 16518$ & \\
\hline WW COD $\left(\mathrm{mg} \mathrm{COD} \cdot \mathrm{L}^{-1}\right)$ & $560 \pm 64$ & $618 \pm 185$ & $564 \pm 182$ & $541 \pm 188$ \\
\hline Total influent COD (mg COD $\cdot \mathrm{L}^{-1}$ ) & & $797 \pm 205$ & $989 \pm 206$ & \\
\hline FW S-SO ${ }_{4}\left(\mathrm{mg} \mathrm{S} \cdot \mathrm{L}^{-1}\right)$ & & $224 \pm 96$ & $140 \pm 55$ & \\
\hline $\mathrm{WW} \mathrm{S}-\mathrm{SO}_{4}\left(\mathrm{mg} \mathrm{S} \cdot \mathrm{L}^{-1}\right)$ & $98 \pm 26$ & $114 \pm 13$ & $123 \pm 16$ & $124 \pm 19$ \\
\hline Total influent S-SO ${ }_{4}\left(\mathrm{mg} \mathrm{S} \cdot \mathrm{L}^{-1}\right)$ & & $114 \pm 15$ & $123 \pm 18$ & \\
\hline 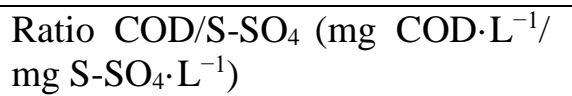 & $5.1 \pm 0.8$ & $7.0 \pm 1.9$ & $8.0 \pm 1.9$ & $5.6 \pm 1.7$ \\
\hline VS reactor $\left(\mathrm{mg} \cdot \mathrm{L}^{-1}\right)$ & $11444 \pm 650$ & $10873 \pm 340$ & $9997 \pm 419$ & $8907 \pm 271$ \\
\hline Total $\mathrm{CH}_{4}\left(\mathrm{~L} \cdot \mathrm{kg}^{-1}\right.$ influent COD $)$ & $49.2 \pm 16.9$ & $110.7 \pm 53.5$ & $144.5 \pm 45.8$ & $89.3 \pm 39.7$ \\
\hline $\begin{array}{l}\text { Increase in methane production } \\
\text { over Period } 1(\%)\end{array}$ & - & $125 \%$ & $193 \%$ & $82 \%$ \\
\hline $\mathrm{SMA}\left(\mathrm{mL} \mathrm{CH} \cdot \mathrm{g}^{-1} \mathrm{VS}\right)$ & $10.44 \pm 0.12$ & $49.22 \pm 0.54$ & $50.98 \pm 0.86$ & $43.59 \pm 0.36$ \\
\hline COD removal by MA* & $111.6 \pm 33.2$ & $266.9 \pm 70.4$ & $345.6 \pm 65.5$ & $210.3 \pm 32.2$ \\
\hline COD removal by sulfate-reducers ${ }^{* *}$ & $320.6 \pm 45.7$ & $274.0 \pm 52.1$ & $214.7 \pm 29.8$ & $290.0 \pm 65.8$ \\
\hline
\end{tabular}

${ }^{*}$ Calculated as g COD transformed into methane $\cdot \mathrm{kg}^{-1}$ influent COD

${ }^{* *}$ Calculated as g COD transformed by sulfate-reducers $\cdot \mathrm{kg}^{-1}$ influent COD

Effluent concentrations in the plant were similar in all periods and lower than the limit concentration allowed to accomplish the discharge requirements $\left(125 \mathrm{mg} \mathrm{COD} \cdot \mathrm{L}^{-1}\right)$, according to Council Directive 91/271/EEC of 21 May 1991. The excellent retention capacity of the membranes made the system capable of achieving high effluent quality in all periods. Regarding membrane fouling, no meaningful differences were observed 
between the different operating conditions when feeding WW in comparison with treating WW jointly with FW.

\subsection{Methanogenic potential in the AnMBR demonstration plant.}

The COD removed during AnMBR operation, besides the COD purged out of the system, can be attributed to two different biological controlled pathways, i.e. (i) sulfate reduction and (ii) methanogenesis. For the COD removed by sulfate-reducers calculation, it was assumed that $2 \mathrm{~g} \mathrm{COD} \cdot \mathrm{g}^{-1} \mathrm{~S}$ reduced are consumed by sulfate-reducers. Contribution of sulfate-reducers to COD removal ranged between 290.0 and $320.6 \mathrm{~g} \mathrm{COD} \cdot \mathrm{kg}^{-1}$ influent COD when only WW was treated and 214.7 and $274.0 \mathrm{~g} \mathrm{COD} \cdot \mathrm{kg}^{-1}$ influent COD in cotreatment periods.

Methane contained in the biogas and dissolved in the effluent was measured daily and used for calculation of the COD removal. The average results for each period are shown in Table 2. Lower yields of COD removal were found in Period 1 due to the characteristically low organic load of urban wastewater. Then, organic load was increased by the addition of FW to the plant influent, while the concentration of sulfates remained stable during the whole period (further details can be found in Moñino et al., 2017). Hence, the observed change in Period 2 and 3 of COD/S-SO 4 ratio (expressed in terms of $\mathrm{mg} \mathrm{COD} / \mathrm{mg} \mathrm{S}-\mathrm{SO}_{4}$ ) is mainly attributed to the addition of this rich source of organic matter (see Table 2). Furthermore, as it has recently been indicated by Paulo et al. (2015), acetoclastic methanogenesis co-exists with the sulfate-reduction of intermediate AD products when influent COD/S-SO 4 ratios are over 3-4 mg COD/mg S-SO 4 . The COD/S$\mathrm{SO}_{4}$ ratio in the AnMBR influent increased to 7 and $8 \mathrm{mg} \mathrm{COD} / \mathrm{mg} \mathrm{S}^{-\mathrm{SO}_{4}}$ due to the FW addition in Periods 2 and 3, respectively. This phenomenon boosted methanogenic pathways in the AnMBR during joint FW and WW treatment: $266.9 \mathrm{~g}$ COD $\mathrm{kg}^{-1}$ influent COD in Period 2 and $345.6 \mathrm{~g} \mathrm{COD} \cdot \mathrm{kg}^{-1}$ influent COD in Period 3 were transformed into 
methane. These results evidence the favourable effect of FW on the whole AD process. The longer the SRT the higher the methane production in AnMBR. The substrate is retained in the system for longer, allowing higher levels of hydrolysis and the consequent increased degradation of slowly biodegradable organic compounds. Under these operational conditions there is a longer contact time between the particulate fraction of the organic matter and the enzymes responsible for its hydrolysis, leading to a higher concentration of hydrolysed products that can be converted into VFAs, which are suitable substrates for MA. Related to this fact, an increase of the measured Specific Methanogenic Activity from 10 to $51 \mathrm{~mL} \mathrm{CH} 4 \cdot \mathrm{g}^{-1} \mathrm{VS} \cdot$ day $^{-1}$ was observed at 42 days and 70 days SRT, respectively (see Table 2). Methane production for each period is also shown in Table 2, which rose to 125 and 193\% in Periods 2 and 3, over Period 1. Nevertheless, the joint treatment of different substrates leads to a synergetic effect, so that, besides the longer SRT, these results suggested the proliferation of a different microbial population while the FW was being added to the AnMBR plant influent. Further experimental support via high-throughput sequencing of 16S rRNA amplicons was therefore required to better understand the influence of FW substrate on AnMBR microbial populations.

\subsection{S rRNA amplicon sequencing}

Illumina sequencing of 16S rRNA amplicons performed in this study allowed a thorough analysis of the microbial community established in the AnMBR. Sludge samples were collected in the four pseudo-steady state periods when VS concentration was stabilised.

A total amount of high quality Illumina reads ranging from 19,321 to 33,556 sequences per sample and $293 \pm 24$ bp mean length were obtained and the taxonomy was assigned within the RDP Classifier tool. After application of a 0.8 confidence-threshold a total amount of 825, 652, 761 and 711 genera in Periods 1,2,3 and 4; was respectively found. 
The percentage of the Archaea or Bacteria genera identified is shown in Table 3. Most of the sequences retrieved were assigned to the Bacteria domain and exceeded $96 \%$ in all the periods, while the remaining sequences belonged to the Archaea domain, reaching a maximum of $3.4 \%$.

Table 3. Relative abundance at domain taxonomic level of the sequences retrieved, sulfate-reducers, methanogenic Archaea (MA) and diversity and evenness indexes in each pseudo steady-state period of the AnMBR.

\begin{tabular}{lcccc}
\hline & Period 1 & Period 2 & Period 3 & Period 4 \\
\hline Archaea (\%) & 0.9 & 2.3 & 3.4 & 3.2 \\
Bacteria (\%) & 99.1 & 97.7 & 96.6 & 96.8 \\
Sulfate-reducers (\%) & 4.6 & 1.5 & 2.8 & 3.1 \\
MA (\%) & 0.5 & 2.2 & 3.0 & 2.9 \\
MA/sulfate-reducers ratio & 0.1 & 1.4 & 1.1 & 1.0 \\
Shannon Index & 3.6 & 3.0 & 3.1 & 3.5 \\
Inverse Simpson Index & 13.7 & 5.8 & 5.9 & 9.2 \\
\hline
\end{tabular}

3.4. Characterisation of microbial population in the AnMBR

A complex and heterogeneous microbial population was characterized in the AnMBR during the joint FW and WW treatment experience. The different Archaea orders and Bacteria phyla detected in the study are shown in Figure 2a and 2b with their relative abundances. As it can be seen in this figure, a shift in the microbial population in the AnMBR appeared as the FW was being added to the influent (Period 2). The main difference with respect to Period 1 was found in the composition of the methanogenic population, as it changed from a heterogeneous combination of acetoclastic, hydrogenotrophic and or methylotrophic methanogens to a community dominated by acetoclastic-capable Archaea orders like Methanosarcinales. 
$\square \quad 0-1 \% \quad \square \quad 1-10 \% \quad \square \quad 10-20 \% \quad \square \quad 20-30 \% \quad \square \quad 30-40 \% \quad \square \quad 40-50 \% \quad \square \quad 50-80 \% \quad \square \quad 80-100 \%$

(a)

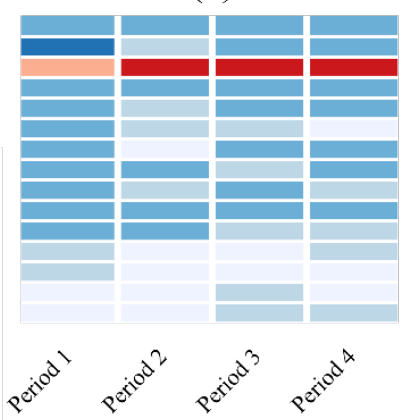

(b)

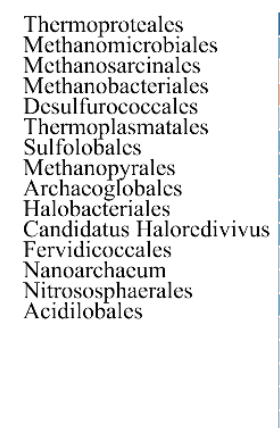

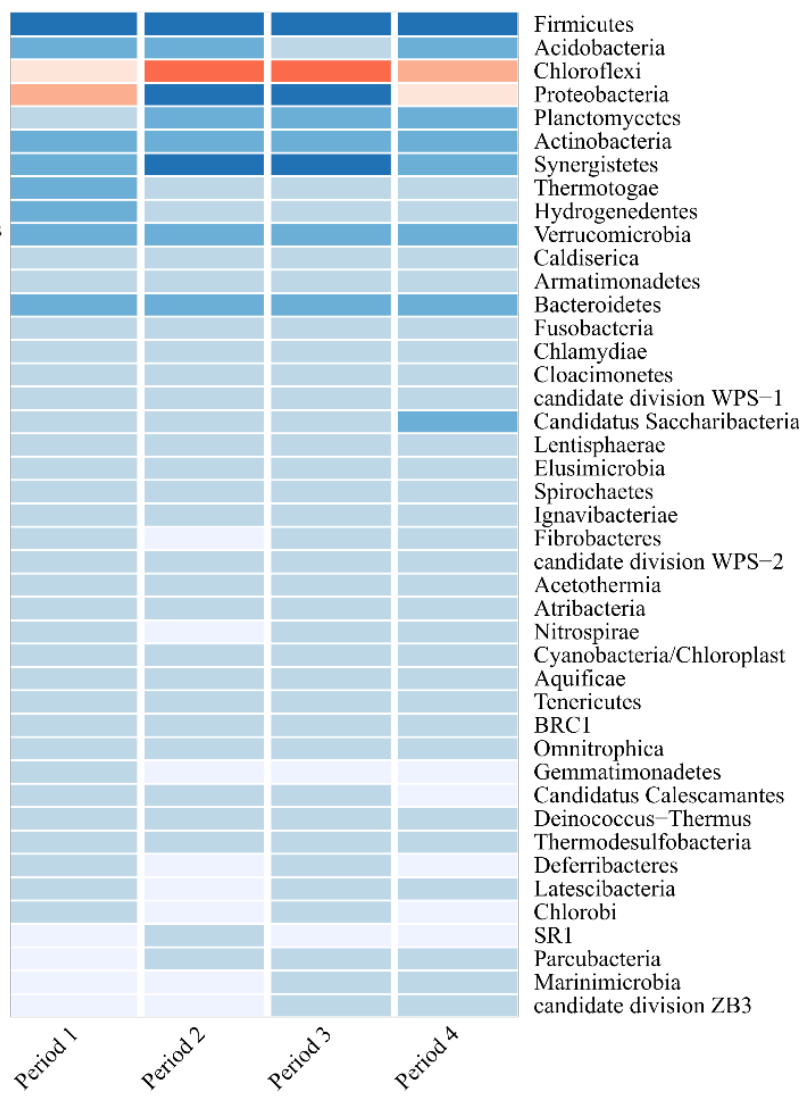

Figure 2. Heatmap showing the composition of microbial community at (a) Archaea order and (b) Bacteria phyla taxonomic levels.

\subsubsection{Methanogenic Archaea}

Methane production in $\mathrm{AD}$ relies on the activity of the methanogenic Archaea (MA) population. These microorganisms grow on a narrow spectra of substrates like $\mathrm{H}_{2}$ and $\mathrm{CO}_{2}$ or formate (hydrogenotrophic methanogenic Archaea, $\mathrm{H}_{2} \mathrm{MA}$ ), although some MA can also reduce a wider spectra of substrates to methane, including here acetate (acetoclastic methanogenic Archaea, AcMA) or methylated compounds (methylotrophic methanogenic Archaea, MeMA) (Lyu and Lu, 2015). Most of the methanogenic orders detected in the AnMBR were $\mathrm{H}_{2} \mathrm{MA}$ (Methanobacteriales, Methanopyrales, Methanomicrobiales and some Methanosarcinales), although some AcMA belonging to the Methanosarcinales order were observed (Table S1). In Period 1, a heterogeneous 
community, slightly dominated by Methanosarcinales (34.0\% of total MA) and Methanomicrobiales (13.6\%) was detected. In the joint FW and WW treatment period a change in MA population took place. The Methanosarcinales order was clearly favoured, from $34.0 \%$ in Period 1 to $87.1 \%$ in Period 2. The relative abundance of this order, in which $\mathrm{H}_{2} \mathrm{MA}$ and AcMA have been reported, remained at values over $82.0 \%$ in the subsequent pseudo steady-state periods analysed.

The Methanosarcinales order contains AcMA genera such as Methanosaeta, whose relative abundance, considering the total amount of genera (including Bacteria and Archaea) increased from 0.2 in Period 1 to $1.9 \%, 2.7 \%$ and 2.5\% in Periods 2, 3 and 4, respectively. In Figures 3a and 3b, the relative abundance of Archaea and Methanosaeta is shown, describing a proportional increase. It therefore seems that the increase in Archaea is due to the notable relative increase in the Methanosaeta genus from $57.0 \%$ to percentages higher than $82 \%$ (Table S1). The enrichment of this genus in the AnMBR may also explain the increased SMA values recorded in Periods 2 and 3 during joint FW and WW treatment (see Table 2). Di Maria and Barrata (2015) also detected high relative abundance of this genus when co-digesting sludge with food wastes (mainly composed of potato, fruit and vegetables). Methanosaeta was also dominant in a recently reported study on anaerobic waste food digestion in a mesophilic reactor (Zamanzadeh et al., 2016).

Relative abundances of relevant genera with respect to the total amount of sequences (including both Archaea and Bacteria domains) were calculated for trend comparison to relevant operational parameters such as SMA and COD removal and shown in Figure 3a, $3 \mathrm{~b}$ and 3c. 
(a)

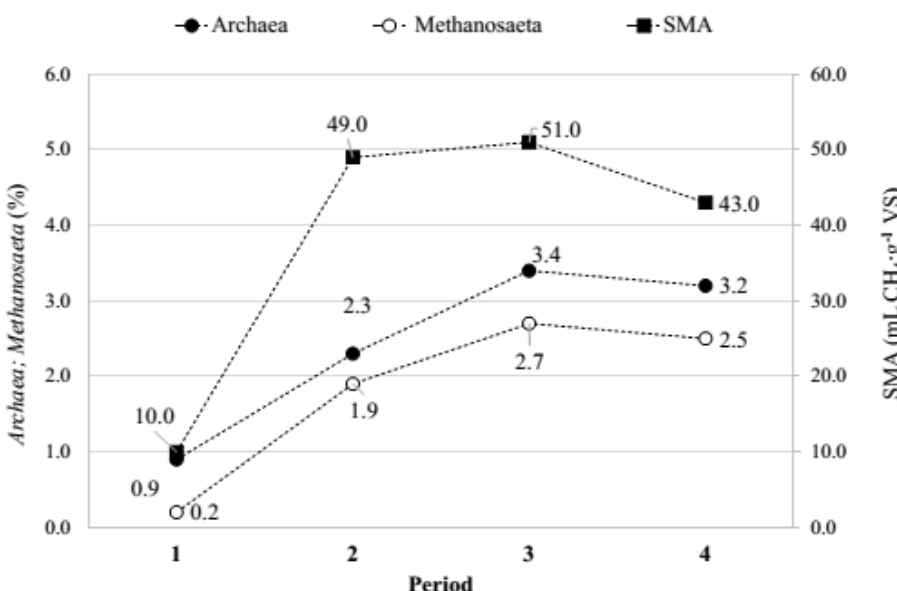

(b)

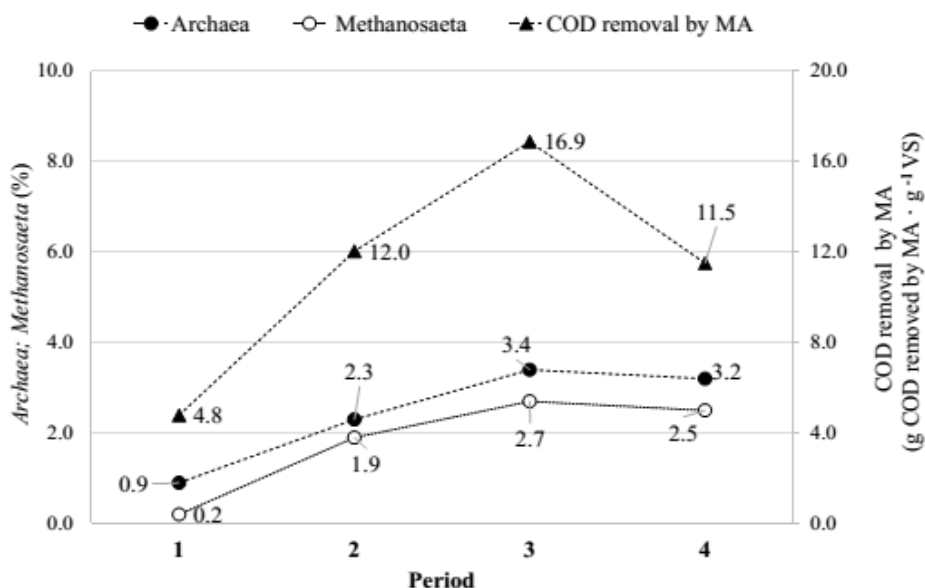

(c)

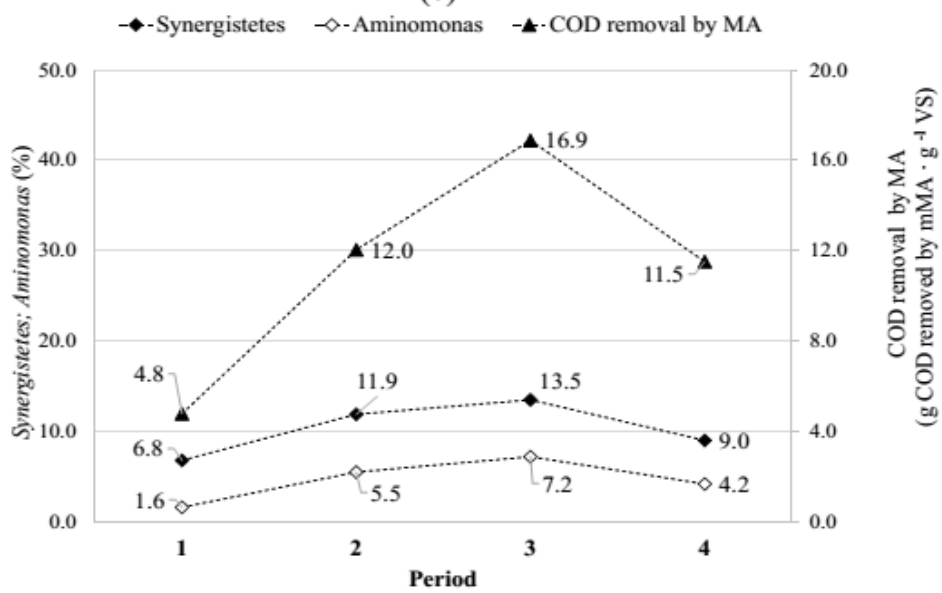

Figure 3. Evolution of: (a) relative abundance of Archaea and Methanosaeta related to SMA values or (b) COD removal by MA, and (c) relative abundance of Synergistetes phylum and related-genus Aminomonas with respect to COD removal by MA. Relative abundances are referred to the total amount of sequences retrieved. 
Comparing the Archaea and Methanosaeta percentages of relative abundance with the SMA (Figure 3a), the same trend can be observed between the relative abundance of Methanosaeta and SMA values, suggesting the implication of this genus in the increased biomethanization capacity of the system. In Figure 3b, the same percentages are plotted against COD removal by MA. According to this figure, proportional increases of both COD removal and Methanosaeta relative abundance can be observed from Period 1 to Period 2 and subsequently to Period 3. However, these trends differ in Period 4, when COD removal starts to fall. In this case, the biodegradability of the influent decreased once FW addition had finished and interfered in the AD hydrolysis and fermentation stages.

The conditions established in the AnMBR system seem to be favourable to the last AD step, as the considerable increase in methanogenic activity with $44 \mathrm{~mL} \mathrm{CH}_{4} \cdot \mathrm{g}^{-1} \mathrm{VS}$ is maintained in Period 4. The advantages of membrane technology should be considered here, as the total retention of the biomass achieved in AnMBR systems also allows suspended microorganisms to remain in the digester, unlike the gravity separation systems. In this study, the addition of FW substrate first enhanced the population's key methanogenic microorganisms, such as those belonging to Methanosarcinales order. Yet, their presence in the system remained stable in the last pseudo steady-state period, after the FW addition experience and more than 70 days of operation, explaining the remarkable methanogenic activity obtained. Further studies on important functional genes in methanogenesis, such as the mcrA gene (Alvarado et al., 2014), may be able to link the relative abundance and role of Methanosaeta and other MA to the higher values of SMA observed (see Table 2).

\subsubsection{Bacteria}

Community analysis of AnMBR sludge samples revealed a major composition of 16 
Bacteria belonging to four main phyla: Chloroflexi, Firmicutes, Synergistetes and Proteobacteria. This bacterial group can explain itself almost $80 \%$ of the microbial diversity detected in each period. Table S1 details the genera composition of each relevant phylum, according to their relative abundance and considering the total amount of genera identified for each phylum in the AnMBR. Only phyla that represented at least $10 \%$ of the bacterial community in any period are given in this table and have been thoroughly explored.

Chloroflexi microorganisms have been widely detected in mesophilic anaerobic digesters (Di Maria and Barratta, 2015; Sundberg et al., 2013; Yi et al., 2014). In the present study, these bacteria seem to play an important role in the anaerobic digestion of FW, as their presence was the highest detected in Periods 2 and 3, when $40 \%$ and $80 \%$ PF of FW was digested together with WW: $41.2 \%$ and $41.3 \%$ of relative abundance of the Bacteria domain, respectively. The most abundant Chloroflexi genus was Levilinea, a mesophilic microorganism that has been reported as a common fermenter from a variety of substrates like sugars and peptides (Yamada et al., 2006). The relative abundance of Levilinea dropped slightly in Periods 2, 3 and 4 to approximately 64\% of the relative abundance in Period 1 (70.4\%). Competition against other Chloroflexi phylum members (Bellilinea or Longilinea) may explain this minor difference. This enhancement of bacteria belonging to the Chloroflexi phylum is in agreement with the previously reported study by Di Maria and Barratta (2015), in which Longilinea was also detected during the mesophilic anaerobic co-digestion of sludge with fruit and vegetable waste. In this study, a high percentage of occurrence of cellulose-containing wastes was also found. A recent study reveals the ability of Anaerolineae class microorganisms, such as Bellilinea and other bacteria classified in the Chloroflexi phylum, to attach to cellulose, which leads to a competitive advantage in complex environments (Xia et al., 2016). According to the 
present study, the dominance of Chloroflexi microorganisms capable of cellulose degradation may have boosted COD removal during joint FW and WW treatment.

Diverse genera belonging to the Firmicutes phylum were also detected in the AnMBR sludge samples. Genus Coprothermobacter suffered a remarkable fourfold decrease after the addition of FW in Period 2. This genus is reported to participate in fermentation pathways in which hydrogen is released (Sun et al., 2015) and may be syntrophically linked to $\mathrm{H}_{2} \mathrm{MA}$. Clostridium is another important genus that belongs to Firmicutes and is commonly found in anaerobic digesters. Species belonging to this genus widely produce acetate as a main sub-product during their fermentation pathways (Yutin and Galperin, 2013). Once again, a link between the fermentation stage by acidogenic bacteria and the acetoclastic pathway that characterizes the Methanosaeta genus seems to be related. Moreover, this relationship also links to the higher SMA observed in Periods 2 and 3.

Less abundant genera belonging to the Firmicutes phylum, like Garciella and Lactobacillus, should also be considered, due to their involvement in the fermentation stage of carbohydrates and more complex substrates. Indeed, Lactobacillus has been reported to degrade cellulose-related products (Sträuber et al., 2016). However, its relative abundance in the AnMBR was only noticeable in Period 4, suggesting that this genus was surpassed by other microorganisms with a higher affinity to the FW composition, like the Chloroflexi phylum. However, when only treating WW a slight fluctuation in microbial population was detected, Lactobacillus was the dominant genus in the Firmicutes phylum.

The phylum Synergistetes was also significantly abundant in the AnMBR throughout the experimental period, exhibiting values close to $10 \%$ of the total Bacteria detected. The aminoacid degradation capacity of microorganisms belonging to the Synergistetes 18 
phylum has been previously reported by Hugenholtz et al. (2009). Related to this phylum, Aminiphilus, Aminobacterium and Aminomonas genera were detected in the AnMBR. The Aminomonas microorganisms thrived during the joint FW and WW treatment and were the most abundant Synergistetes genus, whose relative abundance rose to $55.4 \%$ in Period 3, when the maximum amount of FW was being treated in the plant. The higher biodegradability of the influent in Periods 2 and 3 (due to the FW), might promote the hydrolysis of proteins with the consequent release of aminoacids, which were finally fermented into small carbon compounds and VFAs by Synergistetes belonging microorganisms. This phenomenon seems to explain the similar trend shown in Figure 3c, which plots COD removal by MA against the relative abundance of this phylum and the dominant Aminomonas genus. The release of these fermentation by-products seems to also favour the methanogen population, thus supporting the noticeable enhancement of SMA values and COD removal in terms of methane production in Periods 2 and 3.

The Proteobacteria relative abundance fell during joint FW and WW treatment. As this is a widely diverse taxonomic group, the microorganisms belonging to it are involved in different metabolic reactions. Their suitability for the degradation of polysaccharides has recently been reported when operating an AnMBR treating cellulose-enriched sewage (Watanabe et al., 2016). The simultaneous presence of Proteobacteria and Chloroflexi phyla may therefore play an important role in FW degradation. Proteobacteria microorganisms are not only involved in AD hydrolysis and fermentation steps, but also in the degradation of intermediate products using sulfate, sulphite or thiosulfate as electron acceptors (El Fantroussi et al., 1997), which are present in the AnMBR plant influent.

3.5. Co-existence of sulfate-reducing microorganisms with methanogens in the AnMBR Even when high concentrations of sulfate in the influent are found, acetoclastic 19 
methanogenesis can prevail despite of the suitable pathway of VFAs degradation by sulfate reducers. As mentioned in Section 3.2, the COD/S-SO 4 ratio was enhanced during the joint treatment periods due to the FW addition. This change boosted methanogenic metabolic pathways as it was also observed in terms of COD removal (Table 2).

Different known sulfate-reducers, such as Desulfurococcales, Sulfolobales (Archaea domain), Desulfarculales, Desulfobacterales, Desulfovibrionales, Desulfobacteriales, Desulfuromonadales, Thermodesulfobacterales and Desulfaculales (Bacteria domain), were detected in the AnMBR. The relative abundance percentage of these sulfate-reducer orders was compared to MA orders, like Methanobacteriales, Methanomicrobiales, Methanopyrales and Methanosarcinales (see Table 3). The Desulfomonile relative abundance rose from $1.0 \%$ in Period 1 to $14.2 \%$ in Period 2 and remained at similar relative abundance values inside this phylum, being the dominant sulfate reducer genus identified in the AnMBR. The MA/sulfate-reducers ratio was calculated and revealed a considerable rise in Periods 2 and 3, coinciding with FW addition. Hence, in the AnMBR plant the co-existence of AcMAs like Methanosaeta with sulfate-reducers was observed, not only contributing to high removal of COD but also to high biomethanization values.

\subsection{Diversity analysis of the AnMBR population}

The richness and evenness of the AnMBR system was estimated by the Shannon-Wienner and Simpson indexes (Table 3). The loss of diversity in the reactor was observed during the joint treatment of FW, according to the similar decreasing trends of both estimations. The common range of the Shannon-Wienner index is between 1.5 and 3.5 and therefore the high values obtained in this study between 3.0 (Period 2) and 3.6 (Period 1), support the huge complexity of the AnMBR. As it is observed in this study, increased SRT provided by membrane technology operation allows the system to retain a widely diverse biomass, increasing the heterogeneity of the system. Estimating the diversity indexes 20 
gives a better understanding of the specialization of the biomass.

Lower values of Shannon-Wiener and inverse Simpson diversity indexes were obtained as a higher fraction of FW was treated in the AnMBR. Evenness of some of the Chloroflexi and Synergistetes phyla also increased during Period 2 and 3. Although advantageous effects of increased SRT might contribute to AnMBR population changes observed, the additional organic matter source, i.e FW; acted as a selection factor over the AnMBR biomass by shaping the microbial community. This trend is strongly supported by the estimation of the inverse Simpson dominance index, whose value decreased as a higher fraction of FW was added to the influent. Finally, when FW was no longer supplied (Period 4), both diversity indexes remained lower than those obtained in Period 1. These results support the long-term establishment of a FW-degrading community in the reactors. Membrane technology allowed the efficient microbial community established during FW treatment to remain in the AnMBR, improving its capacity for AD from urban WW.

\subsection{FW-degrading microbial population in the AnMBR}

In this study the addition of FW to the influent of the AnMBR plant enhanced the SMA of the system and the methane content of the biogas. Accordingly, the substrate composition shifted the AnMBR microbial population in Period 1 to the community detected in Periods 2 and 3, when a different amount of FW was treated. The FWdegrading microbial population was thus established during the joint treatment experience in the AnMBR plant and subsequently remained. Substrate composition and SRT have been reported to strictly control AD microbial communities (Vanwonterghem et al., 2015).

The FW-degrading community detected here has a strong hydrolytic and fermentation potential, which seems to be related to the dominance of the Chloroflexi phylum. The 
presence of genera like Longilinea, Levilinea, Lactobacillus or Garciella, and the higher composition of carbohydrates and complex polysaccharides of FW seem to be closely related (Yi et al., 2014). The fermentation of the hydrolysed protein content in the AnMBR influent during FW treatment was remarkable, due to the spread of more peptide fermenters like those belonging to the Synergistetes phylum such as Aminomonas. An efficient set of fermentation reactions, driven by the microbial community established in the AnMBR, would have led to higher production of key intermediate AD products, such as hydrogen or acetate in the system. The efficient transfer of these by-products, especially when they are reduced to acetate, allowed the dominance of acetoclastic Methanosaeta members inside the MA population, enhancing methanogenic activity in the AnMBR.

Interestingly, the FW-degrading population remained in the AnMBR when this substrate was no longer being added to the plant influent, i.e. in Period 4. Although minor changes in the relative abundance of important FW-degrading phyla were found in Period 4, Chloroflexi and Synergistetes remained at high relative abundance values in the AnMBR. The composition of the microbial population detected in the AnMBR 70 days after stopping the joint treatment (Period 4), strongly supports the reported improvement in the anaerobic treatment of WW in this plant. According to the high SMA obtained (43 vs 10 $\mathrm{mL} \mathrm{CH}{ }_{4} \cdot \mathrm{g}^{-1} \mathrm{VS}$ ) and the $82 \%$ increase in methane production between Periods 1 and 4 (see Table 2), the resulting microbial population found in the AnMBR shows a remarkable potential for high energy recovery from WW. However, further research is needed for a better understanding of their metabolic implications for the AD process. Metaproteomics jointly with metagenomics approaches could reveal important information of the degrading potential of this community, dominated by Chloroflexi, Firmicutes, Proteobacteria and Synergistetes. This would also reveal how the AD 
processes can be improved through the addition of highly biodegradable substrates such as FW during a short period. With this strategy, limiting steps such as hydrolysis are overpassed and, consequently, suitable effects among the whole AD steps finally lead to an efficient conversion of organic matter and a remarkable recovery of energy.

In this study the characterization of microbial composition in an AnMBR was reported after Illumina sequencing of v4 hyper-variable region 16S rRNA amplicons. According to the results obtained, the substrate has a strong influence on microbial population dynamics in anaerobic digesters. Although membrane technology can enhance the diversity of sludge microbial communities, the substrate acts as a selective factor, resulting in specific substrate-degrading communities. This substrate-dependent population is characterized by lower diversity, but has a remarkable effect on urban WW treatments. These significant results should be considered in the future management of anaerobic urban wastes, as they reveal the possibility of boosting microbial populations with rich and easily degradable organic substrates.

The combination of a substrate with a high organic matter content like FW in a digester configuration such as an AnMBR, in which biomass is highly concentrated, produced a selection of microorganisms with a wide ability to efficiently degrade organic matter from different sources. Furthermore, the stability provided by the AnMBR configuration in long-term operations in this pilot-plant allowed the microbial population that had been established to remain in the digester, thus improving the performance in treating urban WW. These results show that monitoring microbial responses to operational conditions is not only necessary for a better understanding of the $\mathrm{AD}$ process but is also essential to improve its management.

\section{Conclusions}

A remarkable change in the original microbial population was detected through Illumina 
16S amplicon sequencing of an AnMBR demonstration plant during joint FW and WW treatment. Phyla with high hydrolytic and fermentation potentials (41.2\%-41.3\% Chloroflexi, 10.8\%-10.8\% Firmicutes, 17.4\%-18.3\% Proteobacteria and 11.9\%-13.5\% Synergystetes) and acetoclastic methanogens like Methanosaeta thrived during joint treatment. The establishment of this population as the FW fraction increased, enhanced the SMA from 10 to $43 \mathrm{~mL} \mathrm{CH}_{4} \mathrm{~g}^{-1} \mathrm{VS}$ and provided a boost of $82 \%$ in methane production. The FW-degrading population was not only established during the joint treatment phase but also remained in the AnMBR, favoured by membrane technology, leading to the observed improvement of the anaerobic treatment of urban WW.

\section{Acknowledgements}

This research work has been financially supported by the Generalitat Valenciana (PROMETEO/2012/029 PROJECT), which is gratefully acknowledged.

\section{References}

Alvarado, A., Montañez-Hernández, L.E., Palacio-Molina, S.L., Oropeza-Navarro, R., Miriam, P., 2014. Microbial trophic interactions and mcr A gene expression in monitoring of anaerobic digesters. Front. Microbiol. 5, 1-14. doi:10.3389/fmicb.2014.00597

APHA, 2005. Standard Methods for the Examination of Water and Wastewater. Stand. Methods 541. doi:10.2105/AJPH.51.6.940-a

Aronesty, E., 2011. Ea-utils : Command-line tools for processing biological sequencing data. Expr. Anal. Durham.

Bartram, A.K., Lynch, M.D.J., Stearns, J.C., Moreno-Hagelsieb, G., Neufeld, J.D., 2011. Generation of multimillion-sequence 16S rRNA gene libraries from complex microbial communities by assembling paired-end Illumina reads. Appl. Environ. Microbiol. 77, 3846-3852. doi:10.1128/AEM.02772-10 
Caporaso, J.G., Lauber, C.L., Walters, W. a, Berg-Lyons, D., Lozupone, C. a, Turnbaugh, P.J., Fierer, N., Knight, R., 2011. Global patterns of 16S rRNA diversity at a depth of millions of sequences per sample. Proc. Natl. Acad. Sci. U. S. A. 108 Suppl, 451622. doi:10.1073/pnas.1000080107

Carballa, M., Regueiro, L., Lema, J.M., 2015. Microbial management of anaerobic digestion: Exploiting the microbiome-functionality nexus. Curr. Opin. Biotechnol. 33, 103-111. doi:10.1016/j.copbio.2015.01.008

Cole, J.R., Wang, Q., Cardenas, E., Fish, J., Chai, B., Farris, R.J., Kulam-Syed-Mohideen, A.S., McGarrell, D.M., Marsh, T., Garrity, G.M., Tiedje, J.M., 2009. The Ribosomal Database Project: Improved alignments and new tools for rRNA analysis. Nucleic Acids Res. 37. doi:10.1093/nar/gkn879

Degnan, P.H., Ochman, H., 2012. Illumina-based analysis of microbial community diversity. Isme J 6, 183-194. doi:10.1038/ismej.2011.74

Di Maria, F., Barratta, M., 2015. Boosting methane generation by co-digestion of sludge with fruit and vegetable waste: Internal environment of digester and methanogenic pathway. Waste Manag. 43, 130-136. doi:10.1016/j.wasman.2015.06.007

Edgar, R.C., Haas, B.J., Clemente, J.C., Quince, C., Knight, R., 2011. UCHIME improves sensitivity and speed of chimera detection. Bioinformatics 27, 2194-2200. doi:10.1093/bioinformatics/btr381

El Fantroussi, S., Mahillon, J., Naveau, H., Agathos, S.N., 1997. Introduction of anaerobic dechlorinating bacteria into soil slurry microcosms and nested-PCR monitoring. Appl. Environ. Microbiol. 63, 806-811. doi:10.1007/978-94-017-17113_37

Fisgativa, H., Tremier, A., Le, S., Bureau, C., Dabert, P., 2017. Understanding the 25 
anaerobic biodegradability of food waste: Relationship between the typological , biochemical and microbial characteristics. J. Environ. Manage. 188, 95-107. doi:10.1016/j.jenvman.2016.11.058

Galib, M., Elbeshbishy, E., Reid, R., Hussain, A., Lee, H.S., 2016. Energy-positive food wastewater treatment using an anaerobic membrane bioreactor (AnMBR). J. Environ. Manage. 182, 477-485. doi:10.1016/j.jenvman.2016.07.098

Giménez, J.B., Martí, N., Ferrer, J., Seco, A., 2012. Methane recovery efficiency in a submerged anaerobic membrane bioreactor (SAnMBR) treating sulfate-rich urban wastewater: evaluation of methane losses with the effluent. Bioresour. Technol. 118, 67-72. doi:10.1016/j.biortech.2012.05.019

Giménez, J.B., Robles, A., Carretero, L., Durán, F., Ruano, M. V., Gatti, M.N., Ribes, J., Ferrer, J., Seco, A., 2011. Experimental study of the anaerobic urban wastewater treatment in a submerged hollow-fibre membrane bioreactor at pilot scale. Bioresour. Technol. 102, 8799-8806. doi:10.1016/j.biortech.2011.07.014

Hugenholtz, P., Hooper, S.D., Kyrpides, N.C., 2009. Focus: Synergistetes: Genomics update. Environ. Microbiol. 11, 1327-1329. doi:10.1111/j.1462-2920.2009.01949.x

Kujawa-Roeleveld, K., Elmitwalli, T., Zeeman, G., 2006. Enhanced primary treatment of concentrated black water and kitchen residues within DESAR concept using two types of anaerobic digesters. Water Sci. Technol. 53, 159-168. doi:10.2166/wst.2006.265

Lyu, Z., Lu, Y., 2015. Comparative genomics of three Methanocellales strains reveal novel taxonomic and metabolic features. Environ. Microbiol. Rep. 7, 526-537. doi:10.1111/1758-2229.12283

Mao, C., Feng, Y., Wang, X., Ren, G., 2015. Review on research achievements of biogas 26 
from anaerobic digestion. Renew. Sustain. Energy Rev. 45, 540-555. doi:10.1016/j.rser.2015.02.032

Mattsson, J., Hedström, A., Ashley, R.M., Viklander, M. 2015. Impacts and managerial implications for sewer systems due to recent changes to inputs in domestic wastewater - A review. J. Environ. Manage. 161, 188-197. doi:10.1016/j.jenvman.2015.06.043.

Moñino, P., Aguado, D., Barat, R., Jiménez, E., Giménez, J.B., Seco, A., Ferrer, J., 2017. A new strategy to maximize organic matter valorization in municipalities: combination of urban wastewater with kitchen food waste and its treatment with AnMBR technology. Submitt. to Waste Manag. doi:doi.org/10.1016/j.wasman.2017.02.006

Moñino, P., Jiménez, E., Barat, R., Aguado, D., Seco, A., Ferrer, J., 2016. Potential use of the organic fraction of municipal solid waste in anaerobic co-digestion with wastewater in submerged anaerobic membrane technology. Waste Manag. 56, 158165. doi:10.1016/j.wasman.2016.07.021

Oksanen, J., Blanchet, F.G., Friendly, M., Kindt, R., Legendre, P., McGlinn, D., Minchin, P.R., O’Hara, R.B., Simpson, G.L., Solymos, P., Stevens, M.H.H., Szoecs, E., Wagner, H., 2016. Vegan: community ecology package. R package version 2.3-1. Cran.

Ozgun, H., Gimenez, J.B., Ersahin, M.E., Tao, Y., Spanjers, H., van Lier, J.B., 2015. Impact of membrane addition for effluent extraction on the performance and sludge characteristics of upflow anaerobic sludge blanket reactors treating municipal wastewater. J. Memb. Sci. 479, 95-104. doi:10.1016/j.memsci.2014.12.021

Paulo, L.M., Stams, A.J.M., Sousa, D.Z., 2015. Methanogens, sulfate and heavy metals: 
a complex system. Rev. Environ. Sci. Biotechnol. doi:10.1007/s11157-015-9387-1

Schmieder, R., Edwards, R., 2011. Quality control and preprocessing of metagenomic datasets. Bioinformatics 27, 863-864. doi:10.1093/bioinformatics/btr026

Smith, A.L., Skerlos, S.J., Raskin, L., 2015. Membrane biofilm development improves COD removal in anaerobic membrane bioreactor wastewater treatment. Microb. Biotechnol. 8, 883-894. doi:10.1111/1751-7915.12311

Sträuber, H., Lucas, R., Kleinsteuber, S., 2016. Metabolic and microbial community dynamics during the anaerobic digestion of maize silage in a two-phase process. Appl. Microbiol. Biotechnol. 100, 479-491. doi:10.1007/s00253-015-6996-0

Sun, L., Pope, P.B., Eijsink, V.G.H., Schn??rer, A., 2015. Characterization of microbial community structure during continuous anaerobic digestion of straw and cow manure. Microb. Biotechnol. 8, 815-827. doi:10.1111/1751-7915.12298

Sundberg, C., Al-Soud, W. a., Larsson, M., Alm, E., Yekta, S.S., Svensson, B.H., Sørensen, S.J., Karlsson, A., 2013. 454 Pyrosequencing Analyses of Bacterial and Archaeal Richness in 21 Full-Scale Biogas Digesters. FEMS Microbiol. Ecol. 85, 612-626. doi:10.1111/1574-6941.12148

Tan, G.Y.A., Lee, P.H., Shih, K., 2016. One for all, and all for one: Exploiting microbial mutualism for a new renaissance in anaerobic digestion. Waste Manag. 53, 1-2. doi:10.1016/j.wasman.2016.05.029

Vanwonterghem, I., Jensen, P.D., Ho, D.P., Batstone, D.J., Tyson, G.W., 2014. Linking microbial community structure, interactions and function in anaerobic digesters using new molecular techniques. Curr. Opin. Biotechnol. 27, 55-64. doi:10.1016/j.copbio.2013.11.004 
Vanwonterghem, I., Jensen, P.D., Rabaey, K., Tyson, G.W., 2015. Temperature and solids retention time control microbial population dynamics and volatile fatty acid production in replicated anaerobic digesters. Sci. Rep. 5, 8496. doi:10.1038/srep08496

Vrieze, J. De, Plovie, K., Verstraete, W., Boon, N., 2015. Co-digestion of molasses or kitchen waste with high-rate activated sludge results in a diverse microbial community with stable methane production. J. Environ. Manage. 152, 75-82. doi:10.1016/j.jenvman.2015.01.029

Watanabe, R., Nie, Y., Takahashi, S., Wakahara, S., Li, Y.-Y., 2016. Efficient performance and the microbial community changes of submerged anaerobic membrane bioreactor in treatment of sewage containing cellulose suspended solid at $25{ }^{\circ} \mathrm{C}$. Bioresour. Technol. 216, 128-134. doi:10.1016/j.biortech.2016.05.049

Wilkins, D., Lu, X.Y., Shen, Z., Chen, J., Lee, P.K.H., 2015. Pyrosequencing of mcrA and archaeal 16s rRNA genes reveals diversity and substrate preferences of methanogen communities in anaerobic digesters. Appl. Environ. Microbiol. 81, 604613. doi:10.1128/AEM.02566-14

Xia, Y., Wang, Y., Wang, Y., Chin, F.Y.L., Zhang, T., 2016. Cellular adhesiveness and cellulolytic capacity in Anaerolineae revealed by omics-based genome interpretation. Biotechnol. Biofuels 9, 111. doi:10.1186/s13068-016-0524-z

Yamada, T., Sekiguchi, Y., Hanada, S., Imachi, H., Ohashi, A., Harada, H., Kamagata, Y., 2006. Anaerolinea thermolimosa sp. nov., Levilinea saccharolytica gen. nov., sp. nov. and Leptolinea tardivitalis gen. nov., sp. nov., novel filamentous anaerobes, and description of the new classes Anaerolineae classis nov. and Caldilineae classis nov. in the . Int. J. Syst. Evol. Microbiol. 56, 1331-1340. doi:10.1099/ijs.0.64169-0 
Yi, J., Dong, B., Jin, J., Dai, X., 2014. Effect of increasing total solids contents on anaerobic digestion of food waste under mesophilic conditions: Performance and microbial characteristics analysis. PLoS One 9. doi:10.1371/journal.pone.0102548

Yutin, N., Galperin, M.Y., 2013. A genomic update on clostridial phylogeny: Gramnegative spore-formers and other misplaced clostridia. Env. Microbiol 15, 26312641. doi:10.1111/1462-2920.12173

Zamanzadeh, M., Hagen, L.H., Svensson, K., Linjordet, R., Horn, S.J., 2016. Anaerobic digestion of food waste - effect of recirculation and temperature on performance and $\begin{array}{llll}\text { microbiology. } & \text { Water } & \text { Res. } & \text { 96, }\end{array}$ doi:http://dx.doi.org/10.1016/j.watres.2016.03.058 